$2^{\circ}$ On prélève $20 \mathrm{~mm}^{3}$ (ou 20,8 ) de lait colorié qu'on étale sur une surface de $480 \mathrm{~mm}^{2}$ (ou $500 \mathrm{~mm}^{2}$ ) tracée sur le porte-objet. On laisse sécher à I'abri de poussière.

$3^{\circ}$ On porte l'immersion à l'huile $1 / 12$ sur la préparation et on examine 10-50 champs suivant que les bactéries sont plus ou moins régulièrement dispersées. On compte chaque élément d'une chaînette de streptocoques. Les places où le lait est étalé irrégulièrement ne seront pas prises en considération;

$4^{\circ}$ On additionne les nombres des germes trouvés dans chaque champs et on divise le total par le nombre de champs examinés (10 à 50 champs). Suivant que le comptage de germes se faisait dans le petit ou dans le grand carré ou encore dans le cercle, le résultat sera multiplié par 100 millions, 10 millions ou 1 million.

En faisant plusieurs préparations à la fois on arrive à analyser un échantillon de lait en 5-8 minutes.

(à suivre).

\title{
QUELQUeS TRAVAUX DE L'OFFice AGRICOLE DE SEINE-ET-OISE
}

\author{
(Fin) \\ par M. MONMIREL, \\ Président de l’Office agricole départemental de Seine-et-Oise
}

\section{V. - ESSAIS DE MACHINES A TRAIRE.}

M. Blanchard, Directeur des services agricoles, frappé par l'emploi de plus en plus généralisé des machines à traire en Belgique et par la nécessité de suppléer à la pénurie de main-d'œuvre a décidé, en 1925, de mettre à l'étude l'emploi rationnel des machines à traire.

A cet effet, l'Office agricole de Seine-et-Oise ouvrit un crédit et des essais furent organisés dans une vacherie de plus de 80 bêtes où l'on put expérimenter deux marques de machines à traire de types différents.

Le contrôle laitier rigoureux exercé dans cette vacherie sur toutes les bêtes de l'exploitation a permis de constituer des lots semblables, donnant la même quantité de lait et de matière grasse. Ce lait provenait de vaches de même poids ayant vêlé à peu près au même moment.

Pour s'assurer de l'homogénéité de deux lots, les bêtes étaient contrôlées chaque jour pendant une période préparatoire et pendant une période finale. L'égalité de rendement pendant ces deux périodes permit d'affirmer qu'une similitude suffisante existait entre les lots et pendant la période intermédiaire d'expérience.

Durant toute la période d'expérience le contrôle du lait fut fait à chaque traite, la traite à la machine du lait en expérience était toujours complétée par une traite à la main qui donnait l'arrière-lait. La présence de cet arrière-lait déterminait la plus ou moins grande valeur mécanique de la machine à traire. Le dosage journalier de la matière grasse et 
l'analyse bactériologique ont permis d'envisager l'influence de la machine à traire sur la production beurrière de la vache et sur la propreté de la traite.

Les expériences de Seine-et-Oise ont été faites dans les étables de Gournay-sur-Marne, exploitées par M. J.-E. LucAs, ingénieur agronome, secrétaire de l'Office agricole, spécialiste des questions de production du lait.

\section{Machine A.}

La machine à traire expérimentée en premier lieu est une machine à succion et compression alternées. Le gobelet trayeur est formé d'un tube trayeur cylindrique en métal à l'intérieur duquel est fixé un cylindre concentrique en caoutchoue. La succion, produite par le vide émanant de la pompe, a pour effet d'amener l'éeoulement du lait. La compression du trayon est produite par l'arrivée et le retrait alternatif de l'air dans la chambre comprise entre le tube métallique et le caoutchouc. La régularité de ces mouvements provient de la pompe. Pour que seuls deux trayons soient soumis en même temps à l'action du vide, il existe un distributeur de pulsations très simple et indéréglable qui reçoit son impulsion directement de la pompe. Ce distributeur renforce également les pulsations et fait que celles-ci deviennent tout à fait nettes.

La compression a trois rôles :

$1^{\circ}$ Elle arrête la succion et donne ainsi un temps de repos dạns la traite.

$2^{\circ}$ Elle décongestionne le trayon en faisant circuler rapidement le sang et le rejetant dans la mamelle.

$3^{\circ}$ Elle masse le trayon et la base de la mamelle, stimulant ainsi la sécrétion des glandes mammaires.

La description détaillée de tous ces appareils fait l'objet de la rédaction des catalogues de maisons et ne rentre pas dans cette étude de contrôle des appareils.

Le rôle de l'Office consiste à noter uniquement les résultats.

Lots de vaches. L'examen des lots de vaches mis en expérience montre la valeur comparative des animaux mis en observation.

TABLEAU A

\begin{tabular}{|c|c|c|c|c|}
\hline $\begin{array}{l}\text { Vaches } \\
\text { No }\end{array}$ & $\begin{array}{l}\text { Production } \\
\text { Lot I }\end{array}$ & $\begin{array}{l}\text { journalière } \\
\text { Lot II }\end{array}$ & Vêlage & Saillie \\
\hline 148 & 10.450 & 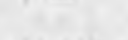 & Février & $4 / 5 / 25$ \\
\hline 144 & & 10.600 & Janvier & $8 / 8 / 25$ \\
\hline 132 & 13 & & Juin & $8 / 8 / 25$ \\
\hline 73 & & 13.200 & Juin & $10 / 6 / 25$ \\
\hline 70 & 10.650 & & Février & $20 / 5 / 25$ \\
\hline 65 & & 9.750 & Février & $26 / 5 / 25$ \\
\hline 152 & 12.950 & & Mars & $28 / 4 / 25$ \\
\hline 88 & & 13.300 & Février & $16 / 5 / 25$ \\
\hline
\end{tabular}


Comme on peut le remarquer, les deux lots étaient sensiblemənt les mêmes tant en ce qui concerne les quantités de lait que l'état même des vaches.

Extraction du lait. L'influence qu'exerce la traite à la machine ressort du tableau ci-après :

\begin{tabular}{|c|c|c|c|c|}
\hline \multirow[b]{3}{*}{ Période préparatoiro } & \multicolumn{2}{|c|}{$\begin{array}{c}\text { TABLEAU B } \\
\text { Lot I } \\
\text { Constamment trait } \\
\text { à la main }\end{array}$} & \multicolumn{2}{|c|}{$\begin{array}{l}\text { Lot II } \\
\text { Trait a la machine } \\
\text { pendant la période d"essai }\end{array}$} \\
\hline & Par lot & Par vache & Par lot & Par vache \\
\hline & 46,850 & 11,71 & 47,050 & 11,76 \\
\hline d'essai ..... & 44,54 & 11,13 & 51,78 & 12,94 \\
\hline finale $\ldots \ldots \ldots \ldots$ & 42,04 & 10,51 & 44,35 & 11,09 \\
\hline
\end{tabular}

On peut admettre que durant la période d'essai, les vaches auraient dû produire la moyenne de lait entre la période préparatoire et la période finale. Le lot témoin, en effet, donne :

$$
\frac{11,71+10,51}{2}=11,11
$$

L’observation montre que le rendement a été de 11,13 , ce qui est naturel.

Le lot d'essai donne : $11,76+11,09$

$$
\overline{2}=11,43
$$

L'observation a permis de noter 12,94, soit une moyenne journalière d'augmentation de 1,5 ce qui est un résultat des plus appréciables.

Ce résultat qui semble surprenant s'explique toutefois par le mode de traite de la machine et par le massage complémentaire de la mamelle à la main. Les recherches sur l'arrière-lait nous ont montré que la traite est opérée à fond par la machine, ce qui est la condition nécessaire pour obtenir un bon rendement constant des glandes mammaires.

Il est, en outre, à remarquer que l'accoutumance a été immédiate et que dès le deuxième jour toutes les vaches ont, pour ainsi dire, donné tout leur lait. Il faut en déduire que la traite à la machine, par sa douceur et sa régularité, n'est pas désagréable aux animaux et qu'elle stimule la sécrétion des glandes mammaires aussi bien que la traite à la main.

Cette sécrétion est accrue aussi par le massage des quartiers de la mamelle qui peut-être fait dans la traite à la main, mais qui, avec la machine, a lieu pendant la traite elle-même, d'où gain de temps.

Arrière-lait. Par la mesure de l'arrière-lait, il est facile de se rendre compte si la traite est opérée à fond.

Sont considérées, en effet, comme traites à fond toutes les vaches ne donnant pas plus de $0 \mathrm{~kg}$. 050 grammes de lait, quantité pouvant souvent être extraite d'une mamelle même après la traite d'un bon vacher. 
Les quantités restant dans la mamelle les dix premiers jours ont été les suivantes :

\begin{tabular}{|c|c|c|c|}
\hline Vache $\mathrm{N}^{0}$ & Maxim, & Minim. & Moyenne \\
\hline 132 & 0 & 0 & 0 \\
\hline 70 & 0,200 & 0 & 0,100 \\
\hline $148 \ldots \ldots \ldots \ldots \ldots \ldots \ldots \ldots$ & 0,300 & 0 & 0,150 \\
\hline $152, \ldots \ldots \ldots \ldots \ldots \ldots \ldots$ & 0,400 & 0,100 & 0,250 \\
\hline
\end{tabular}

Ce tableau montre qu'au bout de dix jours trois vaches sur quatre s'étaient complètement habituées à donner tout leur lait, que, seule, la quatrième $a$, durant tout le cours des essais, maintenu toujours 100 grammes, au moins, dans sa mamelle.

Si nous examinons, parallèlement, la longueur et la grosseur des trayons et si nous attribuons la note 1 aux dimensions les plus petites, la note 4 aux dimensions les plus fortes, chaque bête a un nombre de points d'autant plus faible qu'elle possède de plus petits trayons.

TABLEAU D

\begin{tabular}{|c|c|c|c|c|c|c|c|c|c|c|}
\hline \multirow{2}{*}{$\begin{array}{l}\text { Vaches } \\
\text { No }^{\circ}\end{array}$} & \multicolumn{4}{|c|}{ Trayons antérieurs } & \multicolumn{4}{|c|}{ Trayons postérieurs } & \multirow{2}{*}{$\begin{array}{c}\text { Soumes } \\
\text { des } \\
\text { points }\end{array}$} & \multirow{2}{*}{$\begin{array}{l}\text { Arrière-lait } \\
\text { mogent }\end{array}$} \\
\hline & Diam, & & Long. & & Diam. & 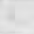 & Iong, & & & \\
\hline 148 & 2,90 & 3 & 8,34 & 3 & 2,37 & 2 & 6,02 & 2 & 10 & 0,150 \\
\hline 132 & 2,07 & 1 & 7,34 & 1 & 2,46 & 3 & 6,26 & 3 & 8 & 0 \\
\hline 70 & 2,39 & 2 & 7,61 & 2 & 2,24 & 1 & 4,96 & 1 & 6 & 0,100 \\
\hline 152 & 3,84 & 4 & 9,35 & 4 & 3,62 & 4 & 9,70 & 4 & 16 & 0,250 \\
\hline
\end{tabular}

En comparant ce classement avec celui de la production d'arrièrelait, il est aisé de constater qu'il existe un véritable parallélisme, les bêtes à gros trayons étant plus longues à s'habituer à la traite à la machine et n'étant pas traites à fond.

Ce fait peut facilement être expliqué. Nous avons, en effet, procédé à la traite mécanique aveo des gobelets et manchons trayeurs d'une seule dimension moyenne, s'adaptant bien aux trayons moyens ou petits, mais dans lesquels les gros trayons s'introduisent difficilement. L'emploi des tubes trayeurs de plus grand diamètre pour les bêtes spéciales semble s'imposer. On peut prévoir des résultats favorables.

Influence sur la matière grasse. Les analyses de matière grasse n'ont pas permis de constater d'influence favorable ou défavorable sur la production beurrière de l'animal.

Vitesse de la traite. Afin de nous rendre compte du rendement dans l'emploi des machines à traire, le temps nécessaire à toutes les manipulations a été soigneusement noté pour chaque traite. 


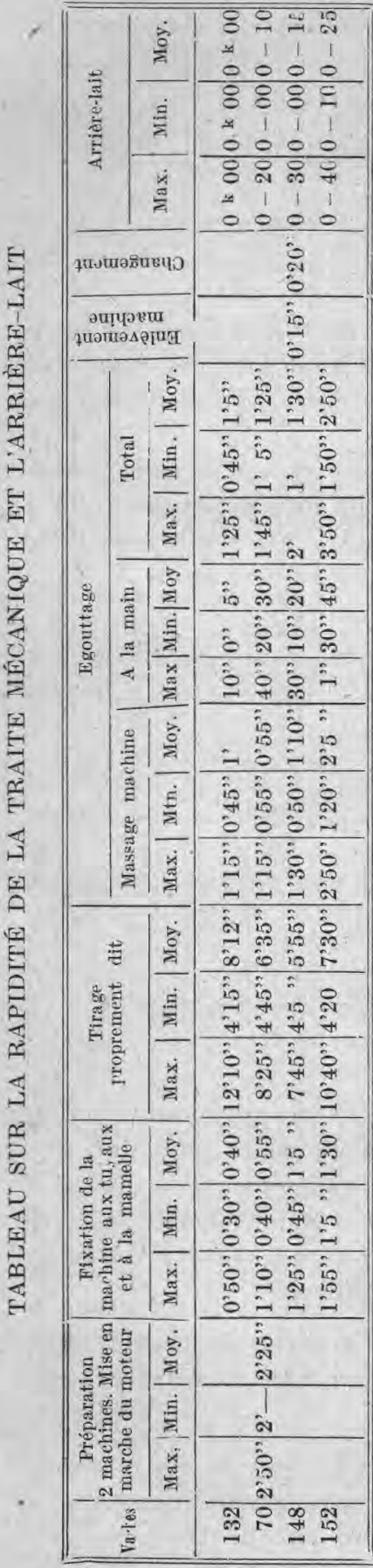

La traite à la main nécessite une moyenne de 5 minutes $20 \mathrm{sec}$. pour une vache donnant cinq litres de laiten moyenne, de 6 minutes 48 sec. pour une vache donnant 7 litres de lait.

Pour la machine à traire, les relevés moyens donnent depuis la pose de la machine au robinet jusqu'à l'enlèvement du tube de caoutchouc du même robinet, y compris le massage, une moyenne de 7 à 8 minutes. Les extrêmes étaient de 6 '65 à $21^{\prime} 65$, ces derniers ayant été réservés aux vaches à gros . trayons.

Le détail des opérations est fixé par le tableau ci-contre :

Ces résultats montrent que la machine à traire, quoique agissant sur les quatre trayons à la fois, est un peu plus lente que la traite à la main. Mais il a été constaté également qu'un même vacher pouvait facilement traire trois vaches à la fois et trouver le temps nécessaire pour procéder au massage de la mamelle.

Un vacher qui soigne 18 bêtes met done, en temps normal, $18 \times 6^{\prime} 48^{\prime \prime}$ soit 2 heures 10 minutes; pendant le même laps de temps, un mécanicien-vacher habile peut traire 40 vaches.

En lui donnant un manœuvre pendant les heures d'alimentation et de nettoyage des animaux, ce mécanicienvacher peut facilement entretenir 40 bêtes. Il apparaît done qu'un homme et une demi-journée d'homme peuvent remplacer deux journées et demi de vacher.

Cette économie d'un tiers sur le temps, de la main-d'œuvre est des plus appréciables dans cette période de pénurie de main-d'œuvre.

Elle nécessite une main-d'œuvre adroite, mais, à proprement parler, 
non spécialisée, ce qui rend possible d'envisager un roulement permettant de donner quelque congé aux vachers.

Propreté de la traite. Les essais au Laboratoire ont permis de suivre les laits tant au point de vue de l'acidité que de l'action de la réductase.

Les résultats sont donnés dans les tableaux suivants :

TABLEAU F ET F'

Le 21 août 1925

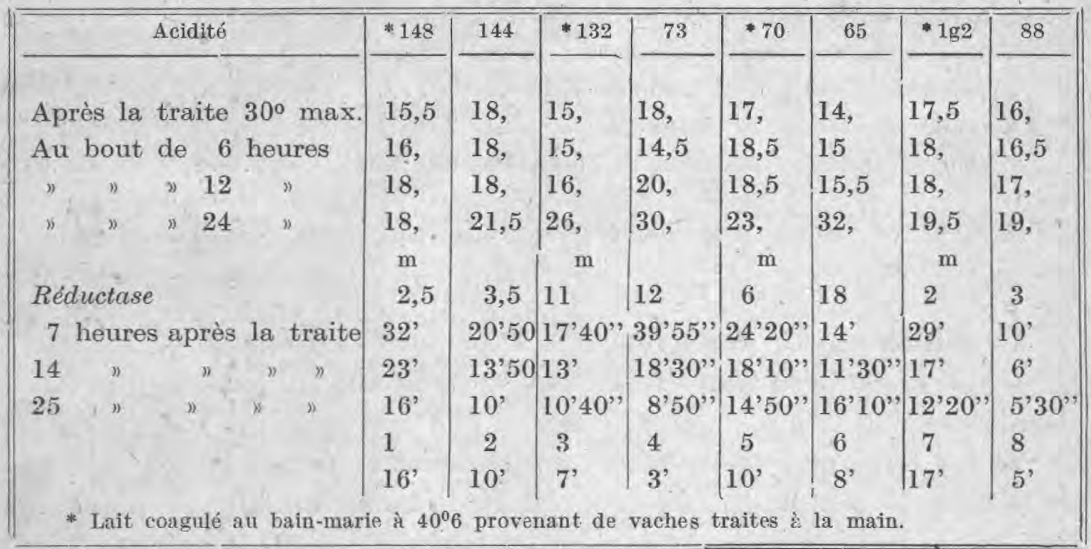

F'

Le 24 août 1925

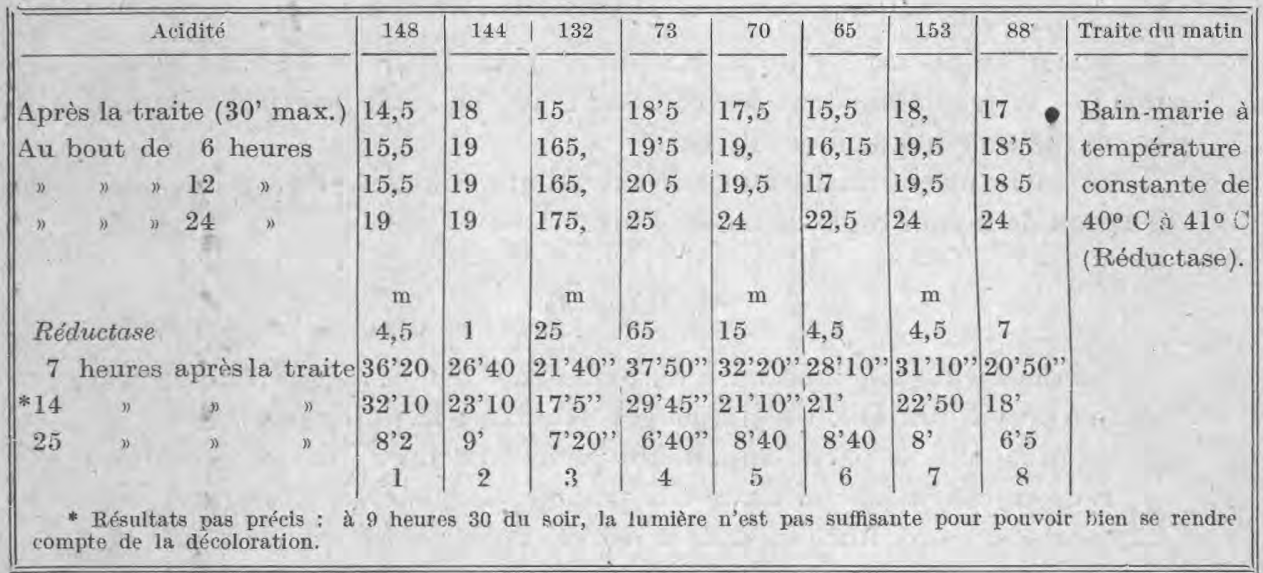

Il semble même que l'acidité du lait soit plus faible pour les laits à la machine et que la réductase soit également légèrement supérieure.

Les méthodes de lavage des appareils étaient celles que recommandent les constructeurs. 
De nouveaux essais avec numération de germes et nettoyages différents des appareils ont été repris au cours de l'été.

\section{RÉSULTATS D'ANALYSES BACTÉRIOLOGIQUES \\ (Essais de Gournay-sur-Marne)}

10 Traite à la main après lavage des avants-bras et des mains au savon, dans un seau ordinaire, ,.....

20 Nettoyage de la machine par simple eirculation d'eau froide et d'eau chaude après chaque traite (Nettoyage après démontage deux fois par semaine)

$3^{\circ}$ Comme ci-dessus, mais circulation d'une solution d'eau de javel à 2 p. 1.000 de chlore libre......

$4^{\circ}$ Démontage et lavage ordinaire comme $2^{\circ}$ après chaque traite (Opération faite par les ouvriers)

Essai I

27.000

308.200

6.750

328.500
Essai II

40.500

337.500

N. B. - Les ensemencements ont tous été effectués six heures après la prise d'échantillon.

Conclusions. De ces premières expériences, il résulte ce qui suit:

$1^{0}$ La machine à traire se montre aussi bonne trayeuse que la main de l'homme.

$2^{\circ}$ La machine à traire massant elle-même et permettant le massage de la mamelle pendant la traite a permis d'obtenir un lait plus abondant que par la traite à la main dans la proportion de $10 \%$ environ.

$3^{\circ}$ La traite mécanique permet d'économiser de la main-d'œuvre, mais revient au point de vue économique aussi cher que la traite à la main.

40 Le simple lavage de la machine à l'eau froide et à l'eau chaude, tel que le recommandent les constructeurs, ne suffit pas pour assurer la production hygiénique du lait.

$5^{\circ}$ Le lavage ordinaire doit être suivi d'une circulation d'eau javellisée à raison de 2 pour mille de chlore libre.

\section{Machine B}

La deuxième machine mise en expérience est basée sur l'utilisation unique de l'aspiration. Un simple gobelet fixé à la tétine par une bague de caoutchoue à sa partie supérieure permet de faire un vide constant.

L'aspiration étant faite par une pompe à air, la dépression est cependant plus ou moins forte suivant la marche de la pompe, ce qui détermine des ondulations de dépression dans le gobelet et maintient sur les tétines une alternance légère rompant l'aspiration constante.

Dans cette expérience, la valeur comparative du lot en expérience et du lot témoin s'établit comme suit: 


\begin{tabular}{|c|c|c|c|c|c|c|}
\hline \multirow{2}{*}{$\begin{array}{l}\text { Numéros } \\
\text { des vaches }\end{array}$} & \multicolumn{2}{|c|}{ Quantités de lait } & \multicolumn{2}{|c|}{ Etat des animaux } & \multirow{2}{*}{\multicolumn{2}{|c|}{ Age }} \\
\hline & Témoin & Expérience & Date du vêlage & Date de la saillie & & \\
\hline 179 & * & 15,6 & $8-11-25$ & & 8 ans & $1 / 2$ \\
\hline 105 & 15,5 & & $13-12-25$ & $20-1-26$ & 7 ans & $1 / 2$ \\
\hline 139 & & 12,1 & $11-9-25$ & $17-2-26$ & & \\
\hline 129 & 11,3 & & $28-9-25$ & $8-1-26$ & & \\
\hline 178 & & 18,4 & $3-10-25$ & & 6 ans & $1 / 2$ \\
\hline 189 & 19,2 & & $16-12-25$ & $30-1-26$ & 8 ans & $1 / 2$ \\
\hline 45 & & 14,4 & $2-10-25$ & $26-11-25$ & 9 ans & \\
\hline 174 & 14,2 & & $1-9-25$ & $1-12-25$ & 6 ans & $1 / 2$ \\
\hline 135 & & 9,9 & $27-9-25$ & $30-11-25$ & 10 ans & $\%$ \\
\hline 93 & 10,2 & & $3-9-25$ & $20-11-25$ & 6 ans & $1 / 2$ \\
\hline 117 & & 7,5 & $6-10-25$ & $4-12-25$ & 12 ans & \\
\hline 166 & 7,9 & - & $25-825$ & $25-11-25$ & 8 ans & \\
\hline & 78,3 & 77,9 & & & & \\
\hline $\begin{array}{l}\text { Moyenne } \\
\text { par vache }\end{array}$ & 13,9 & 12,9 & & & & \\
\hline
\end{tabular}

Comme il est possible de le remarquer, les lots étaient sensiblement les mêmes tant en ce qui concerne la production du lait que l'état des vaches.

Durant le cours de l'expérience, les vaches ont reçu la même nourriture contrôlée.

Extraction du lait. L'influence qu'exerce la traite à la machine ressort du tableau ci-après :

\begin{tabular}{|c|c|c|c|c|c|c|c|c|}
\hline \multirow{3}{*}{ Périodes } & \multirow{3}{*}{$\begin{array}{c}\text { Nombre } \\
\text { de } \\
\text { jours }\end{array}$} & \multicolumn{2}{|c|}{ Quantité lait au kilogr. } & \multirow{3}{*}{$\begin{array}{c}\text { Arrière-lait } \\
\text { compris dans } \\
\text { la qua tité } \\
\text { totale de ait } \\
\text { Litres }\end{array}$} & \multicolumn{2}{|c|}{\begin{tabular}{|l|} 
Quaut, hearr: \\
par ky de I it
\end{tabular}} & \multicolumn{2}{|c|}{$\begin{array}{l}\text { Quantité beurre totale } \\
\text { par lot }\end{array}$} \\
\hline & & Témoin & Expérience & & Tém. & Exp. & Témoin & Expérience \\
\hline & & Litres & Litres & & Grs & Grs & Grs & Grs \\
\hline Préparatoire & 20 & 13 & 12,65 & & 38,5 & 35,8 & 591 & 532,2 \\
\hline & 10 & 13,4 & 12,7 & 1,3 & 39,5 & 32,2 & 624,5 & 482,5 \\
\hline & & 12,5 & 11,6 & 1 & 42 & 38,1 & 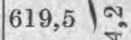 & 521,3 م \\
\hline Hssai & 10 & $\stackrel{\sim}{\sim} 12,1$ & $11,02 \mid \approx$ & 0,72 & 39,6 & 36,7 & 562,1 蛋 & $476,3\}$ \\
\hline & 9 & 12,3 & 11,24 & 0,54 & 39 & 35,2 & 566 & 465,2 \\
\hline Finale & 12 & 11,28 & 11,20 & & 39,5 & 36,1 & 526,8 & 477 \\
\hline
\end{tabular}

On peut admettre que pendant l'expérience les deux lots ont baissé dans la même proportion. En effet, la différence entre eux n'était que de $0 \mathrm{~kg}$. 35 pendant la période préparatoire; pendant la période finale elle était de $0 \mathrm{~kg}$. 08 , ce qui est sensiblement la même chose.

Il est donc permis de supposer qu'au cours des essais, les deux lots 
se sont comportés de la même manière, qu'ils ont diminué dans la même proportion.

La diminution du lot témoin aurait dû être environ celle de la moyenne entre la période préparatoire et la période finale, soit: $10 \times 13+12 \times 11,28$

$$
20+12
$$

0 litre 14 au-dessus de la moyenne.

Celle du lot d'essai aurait dû être de : $20 \times 12,65+12 \times 11,20$

$$
20+12
$$

elle n'a été que de 11,6 , soit une perte de $0 \mathrm{~kg}$. 500, à laquelle il faut ajouter celle de 0,14 de supplément du lot témoin, soit de $0 \mathrm{~kg}$. 64 par vache et par jour.

Si nous examinons les quantités de matière grasse, nous trouvons la perte encore plus sensible. Le lot témoin, dont la moyenne entre la période préparatoire et la période finale est de: $20 \times 591+12 \times 526,8$

32

de 27 gr. 3 par jour, puisque la moyenne de production est de 594,2.

Le lot d'essai, au contraire, dont la moyenne entre la période préparatoire et la période finale est de : $20 \times 532,2+11 \times 477$

$$
20+12
$$

est inférieure de $32 \mathrm{gr}$. 5. La différence dans la production en beurre est donc à l'avantage du lot témoin de $27,3+32,5=59 \mathrm{gr} .8$, soit une perte en beurre atteignant $10 \%$.

II peut paraître 'surprenant que les 640 gr. de lait perdu puissent contenir 59,8 de beurre. Mais il ne faut pas oublier, en effet, que c'est le meilleur lait, le plus riche en matière grasse, celui de la fin qui est retenu par la mamelle. L'influence de l'alimentation, comme le montre le détail du tableau, prouve bien que les oscillations par kilogramme de lait restent parfaitement parallèles durant tout le cours de l'expérience.

Arrière-lait. L'examen des résultats de la traite complémentaire faite après chaque traite à la machine prouve amplement que cette traite gêne la vache et l'empêche de donner tout son lait.

Il est possible de lui extraire une partie de ce lait par la traite à la main. Mais elle en conserve une partie encore, puisque au total, eomme nous l'avons vu, il y a perte de lait.

Comme il ressort du tableau B, la vache s'accoutume petit à petit. Les dix premiers jours, il est possible d'extraire $3 \mathrm{~kg}$. 300 de lait après la traite, les dix jours suivants $1 \mathrm{~kg}$., entre le vingtième et le trentième jour, 0,72, dans les dix derniers jours, 0,54.

Devant des résultats si peu favorables, nous avons cru utile de recher- 
cher le détail des arrières-laits pour chacune des bêtes, en les comparant à la grosseur des trayons pour chacune d'entre elles.

Pour mieux nous rendre compte, nous avons attribué des notes $1,2,3,4,5,6$ à chaeun des trayons en donnant la note 1 aux plus petits, la note 6 aux plus gros. L'addition des quatre notes attribuées ainsi pour la longueur et la largeur des trayons antérieurs et des trayons postérieurs donne pour chaque vache une note qui permet de faire un classement. En classant d'après ces résultats, les bêtes qui ont le chiffre le plus bas sont les animaux aux trayons les plus menus.

\begin{tabular}{|c|c|c|c|c|c|c|c|c|c|c|c|}
\hline \multirow{2}{*}{\multicolumn{2}{|c|}{ Vaches }} & \multicolumn{4}{|c|}{ Trayons antérieurs } & \multirow{2}{*}{\multicolumn{2}{|c|}{ Diamètre }} & \multirow{2}{*}{\multicolumn{2}{|c|}{ Longueur }} & \multirow{3}{*}{$\begin{array}{l}\text { Somme } \\
\text { de } \\
\text { points }\end{array}$} & \multirow{3}{*}{$\begin{array}{l}\text { Arrière-lait } \\
\text { donné pour } \\
\text { chaque bête }\end{array}$} \\
\hline & & \multicolumn{2}{|c|}{ Diamètre } & \multicolumn{2}{|c|}{ Longueur } & & & & & & \\
\hline Race & $\mathrm{N}^{0}$ & Hoyenne & Note & Moyenne & Note & Moyenne & Wote & $\overline{\text { Iloyerne }}$ & Nite & & \\
\hline Normande & 117 & 15 & 1 & 42 & 1 & 16,5 & 1 & 41,5 & 1 & 4 & $0 \mathrm{~kg} 217$ \\
\hline Normande & 45 & 16,5 & 2 & 67,5 & 2 & 17 & 2 & 69,5 & 4 & 11 & $1 \mathrm{~kg} 02$ \\
\hline Hollandaise & 135 & 18 & 3 & 77,5 & 3 & 5,5 & 3 & 65,5 & 3 & 12 & $0 \mathrm{~kg} 075$ \\
\hline Flamande & 139 & 18,5 & 4 & 72,5 & 4 & 20,5 & 4 & 52,2 & 2 & 13 & $0 \mathrm{~kg} 757$ \\
\hline Normande & 178 & 20,5 & 5 & 97 & 5 & 24,5 & 5 & 80 & 6 & 21 & $1 \mathrm{~kg} 40$ \\
\hline Normande & 179 & 25 & 6 & 97,5 & 6 & 29,5 & 6 & 76 & 5 & 23 & $1 \mathrm{~kg} 40$ \\
\hline
\end{tabular}

En comparant ce classement avec celui de la production d'arrière-lait, il est aisé de constater un véritable parallélisme. Les bêtes à gros trayons, étant plus longues à s'habituer à la traite mécanique que les bêtes à petits trayons. Les premières ne sont pas traites à fond.

Une anomalie existe dans ce classement pour la bête normande 45 , qui laisse plus de lait que ne semblait le prévoir son classement. Il faut attribuer ce fait à ce que cette vache avait du vaccin sur les trayons.

La vache hollandaise classée $3^{\text {e }}$ est cependant celle qui donne le moins d'arrière-lait. L'explication peut-être due au fait que cette vache étant de race hollandaise a des trayons plus souples que ceux des vaches normandes.

Pour toutes les autres bêtes, comme dans les autres essais, la méthode apparaît bonne: la grosseur des trayons joue un rôle de premier ordre pour l'accoutumance des vaches à la machine à traire.

Influence sur la matière grasse. Durant les essais, trois fois par semaine les prélèvements de lait ont permis d'étudier les quantités de matière grasse produites, en y comprenant celle due à la production de l'arrière-lait.

Les résultats obtenus ont été notés dans le tableau $B$.

Au total, le lot témoin qui, pendant sa période préparatoire, a donné 591 grammes de beurre et $\mathbf{5 2 6 . 8}$ dans sa période finale, laisse supposer que pendant la période transitoire les bêtes auraient dû donner: 
$20 \times 591+12 \times 526,8$

$$
20+12
$$

594,2 , soit une augmentation de : $594,2-566,8=27,4$.

Le lot en expérience aurait de son côté dû donner :

$532,2 \times 20+12 \times 477$

$$
20+12
$$

soit une perte de $511-478,7=32$ gr. 3 , auxquels il faut ajouter l'absence de gain de 27 gr. 4 , ce qui porte à 27 gr. $4+32$ gr. $3=59$ gr. 7 la perte totale, soit une perte de $10 \%$ dans le rendement.

Le fait que dans cette expérience les vaches ont retenu leur lait a déterminé une perte générale de la matière grasse très appréciable et très préjudiciable au rendement de la bête.

Propreté de la traite. Pour se rendre compte de la valeur de la propreté de la traite, des ensemencements ont été faits comme nous l'avons rappelé au début de ces expériences.

Deux séries d'ensemencement ont été faites en juin 1926 et ont donné les résultats suivants :

Dans la première série, le lait conservé à l'air libre après six heures contenait pour les laits traits à la main 27.500 et 40.700 microbes au centimètre eube; les laits de la machine à traire nettoyés par simple passage d'eau chaude et d'eau froide en contenaient 508.500 et 729.000.

Dans la deuxième série le lait trait à la main contenait dans les mêmes conditions 25.875 microbes; le lait à la machine 42.000 ; le lait à la machine rincé à l'eau javelisée 33.300 et 28.800 .

Pour cette machine également la propreté de la traite ne peut être envisagée qu'en employant des antiseptiques, en particulier l'eau de Javel.

Conclusions. La deuxième machine mise en expérience demande à être mise au point.

Comme pour toutes les machines à traire, il est indispensable de faire des gobelets de plusieurs dimensions, afin de pouvoir les adapter aux trayons de grosseurs différentes.

Il est indispensable aussi d'assurer la régularité danś l'aspiration de la pompe. La nécessité de perfectionnement facile à réaliser a d'ailleurs été retenue par le constructeur.

Les conclusions ayant trait au lavage de la machine et formulées au sujet des essais faits avec la première machine s'appliquent aussi à ces derniers essais. 